\title{
Combined Type II Odontoid Fracture with Jefferson's Fracture Treated with Temporary Internal Fixation
}

\author{
Abhijit Yuvaraj Pawar ${ }^{1}$, Patrick F. O’Leary ${ }^{2}$ \\ ${ }^{1}$ Ruby Hall Clinic and Dr. D.Y. Patil Medical College, Pune, India \\ ${ }^{2}$ Department of Orthopedic Surgery, Hospital for Special Surgery, New York, NY, USA
}

An 18-year-old male presented after a motor vehicle rollover accident. Computed tomography (CT) scan confirmed the diagnosis of Type II odontoid fracture. Considering the patient's young age and the limitations of C1-C2 fusion including significant loss of cervical rotation, temporary internal fixation with a lateral mass fixation of $\mathrm{C} 1$ and pedicle fixation of $\mathrm{C} 2$ without fusion was done. CT scan done at 6-month follow-up visit showed healed odontoid fracture and excellent C1-C2 alignment. At ninth postoperative month, internal fixation was removed. Patient had normal movements of cervical spine at 1-year follow-up. Temporary internal fixation can be an important tool in the armamentarium of the surgeon in treating type II odontoid fractures in young adults and children. This strategy avoids the complications halo fixation and immobilizes the unstable C1-C2 segment without fusion. Removal of the internal fixation after healing allows restoration of the rotational motion.

Keywords: Type II odontoid fractures; Internal fixation; C1-C2 fusion

\section{Introduction}

Odontoid fractures are generally rare and account for approximately $15 \%$ of all cervical fractures [1-3]. In young patients, these fractures are usually secondary to high energy trauma, with motor vehicle accidents being responsible for the majority of odontoid injuries $[1,2,4]$. The most frequent association of type II odontoid fractures is atlas fractures. The incidence of associated atlas and axis fractures is $12 \%$ and in the majority of these cases, the odontoid fracture determines the management strategy [4-6]. Odontoid fractures have been anatomically classified in three types by Anderson and D'Alonzo [7] based on the position of the fracture line. Generally, nonsurgi- cal bracing is the mainstay of management to stable type I and III odontoid fractures and has demonstrated high healing rates $[2,8]$.

The question how to successfully stabilize type II odontoid fractures still remains unanswered. Successful nonsurgical management of type II fractures with halo thoracic vest (HTV) has been reported, however, it is associated with poor patient tolerance and high non-union rate $[1,8]$. As of now, the surgical treatment options include $\mathrm{C} 1-\mathrm{C} 2$ arthrodesis and anterior screw fixation of $\mathrm{C} 2$ and occipitocervical fusion [1,9-12]. Each of these techniques has its own advantages and limitations, however, patient tailored treatment remains the most prominent method for management of these fractures. Odontoid screw fixation

Received Nov 12, 2014; Revised Mar 6, 2015; Accepted Mar 13, 2015

Corresponding author: Abhijit Yuvaraj Pawar

Ruby Hall Clinic and Dr. D.Y. Patil Medical College, Pune 411001, India

Tel: +91-73870-80391, Fax: +91-020-278-05217, E-mail: drabhijitpawar@gmail.com 
preserves atlanto axial rotations, however, this approach is contraindicated in certain fracture orientation and angulation where inter fragmentary compression cannot be achieved $[12,13]$. C1-C2 fusion techniques are successful, however, they are associated with significant loss of rotational motion of the cervical spine $[2,14]$. An alternative treatment option in the form of temporary internal fixation of $\mathrm{C} 1-\mathrm{C} 2$ segment to preserve the motion of $\mathrm{C} 1-\mathrm{C} 2$ segment is described in this case report.

\section{Case Report}

An 18-year-old male presented after a motor vehicle rollover accident. He was taken to the emergency where standard X-ray revealed odontoid fracture. He was transferred to the tertiary care center for further management in a rigid cervical collar. Physical examination revealed significant tenderness in the upper cervical spine. He had restricted neck flexion up to 30 degrees and extended to neutral. He had minimal lateral bending motion and refused to turn head from side to side because of pain. His neurological examination was normal. Anteroposterior (AP), lateral and open mouth odontoid films were obtained followed by computed tomography (CT) scan. CT scan confirmed the diagnosis of Type II odontoid fracture with a gap of $2.5 \mathrm{~mm}$ and anterior displacement of the dens by $4 \mathrm{~mm}$. The fracture angulation was 27 degrees with an associated anterior and posterior arch of atlas fracture leading to unstable left lateral mass of $\mathrm{Cl}$ (Fig. 1). Magnetic resonance imaging (MRI) showed intact transverse ligament. Possible treatment options were nonsurgical management in a halo vest as well as surgical management. Both were discussed with the patient. The patient denied a conservative treatment with halo vest because of the long duration of treatment and high incidence of associated complications.

Since the fracture had angulation of 27 degrees, the surgeon felt that it would be difficult to get appropriate trajectory for screw fixation and in turn adequate reduction. Hence, anterior odontoid screw fixation was not considered. Considering the patient's young age and the limitations of $\mathrm{C} 1-\mathrm{C} 2$ fusion including significant loss of cervical rotation, temporary internal fixation with a lateral mass fixation of $\mathrm{C} 1$ and pedicle fixation of $\mathrm{C} 2$ without fusion was planned. The intention was to remove the internal fixation after radiological healing of odontoid fracture to maintain the rotational motion of the cervical spine. As a part of the preoperative work-up, CT angiogram was done which showed the vertebral artery in an abnormal position and closer to $\mathrm{C} 2$ pedicle (Fig. 2). Hence, the surgeon planned unilateral internal fixation of $\mathrm{C} 1-\mathrm{C} 2 \mathrm{seg}$ ment without fusion. Accordingly, internal fixation of left

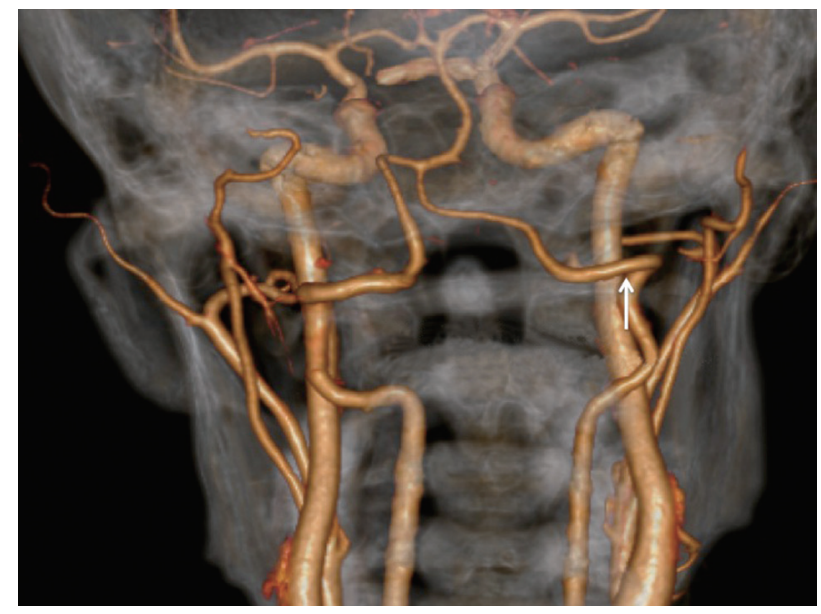

Fig. 2. Vertebral artery close to the pedicle entry point on the right side of C2 (arrow).
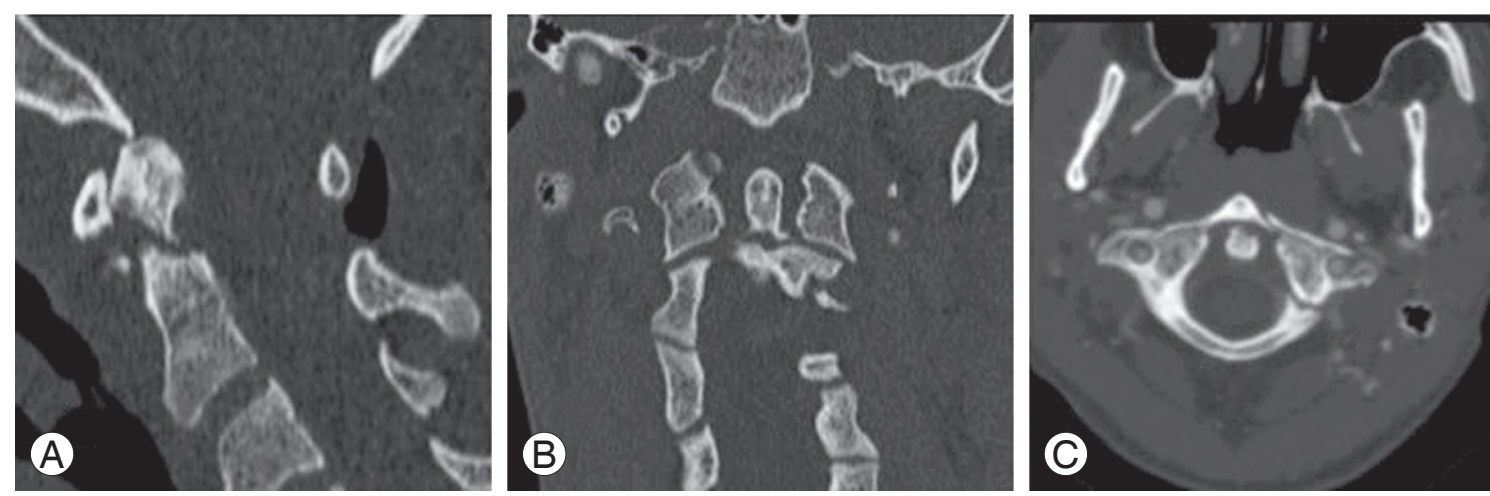

Fig. 1. (A) Type II odontoid fracture with an angulation of about $27^{\circ}$ on sagittal computed tomography scan, (B) coronal view, (C) Jefferson's with unstable left lateral mass of C1. 

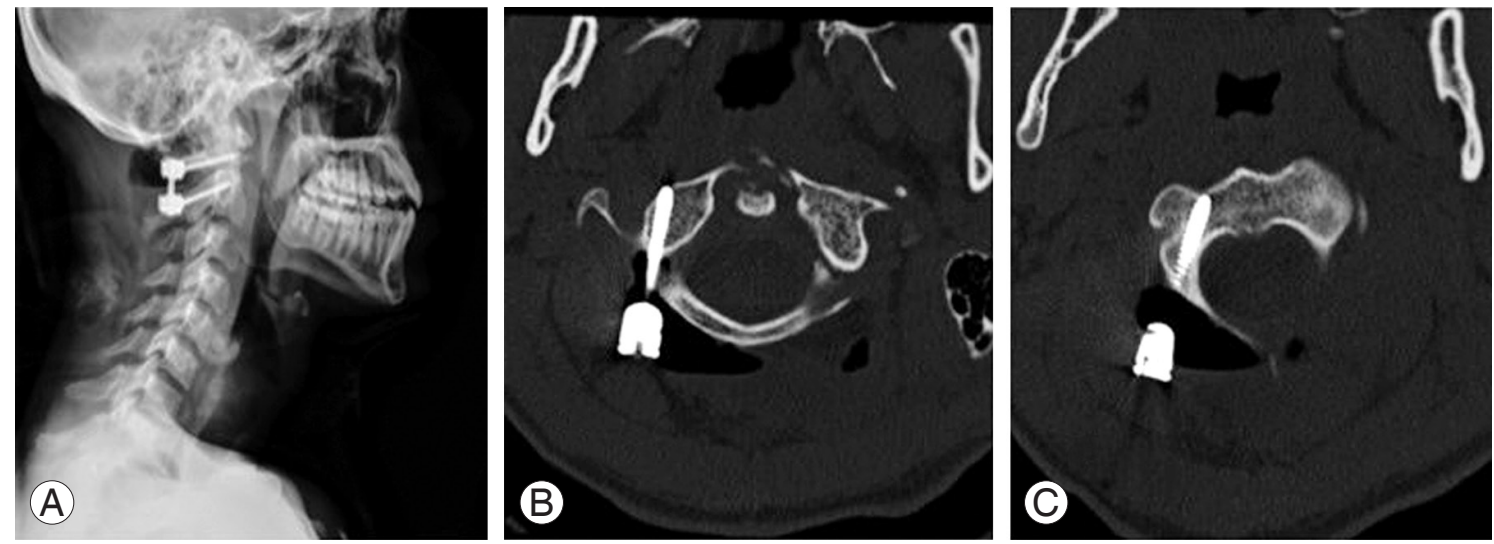

Fig. 3. Postoperative films with internal fixation of C1-C2 segment. (A) C1-C2 internal fixation. (B) Unilateral lateral mass screw fixation in C1. (C) Unilateral pedicle screw fixation in C2.
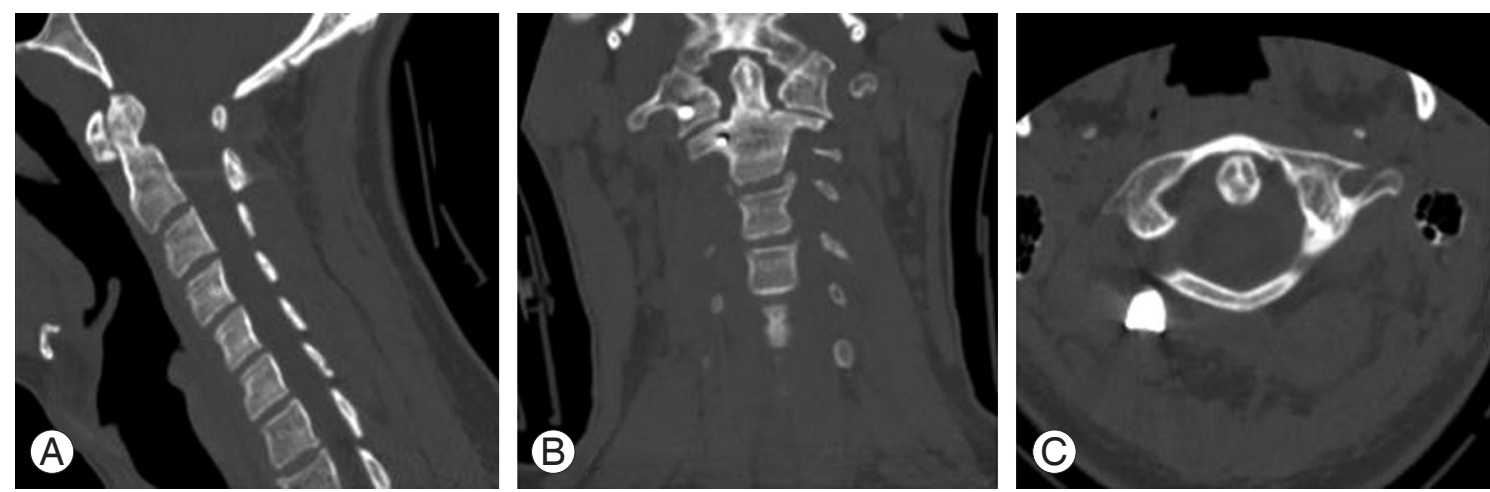

Fig. 4. Follow-up computed tomography scan showing healed type II odontoid fracture and Jefferson's fracture with good alignment. (A) Healed Odontoid fracture in saggital computed tomography (CT) on follow up visit, (B) No fusion of C1-C2 joint on follow up CT, (C) Healed rim fracture of Atlas on follow up CT scan.
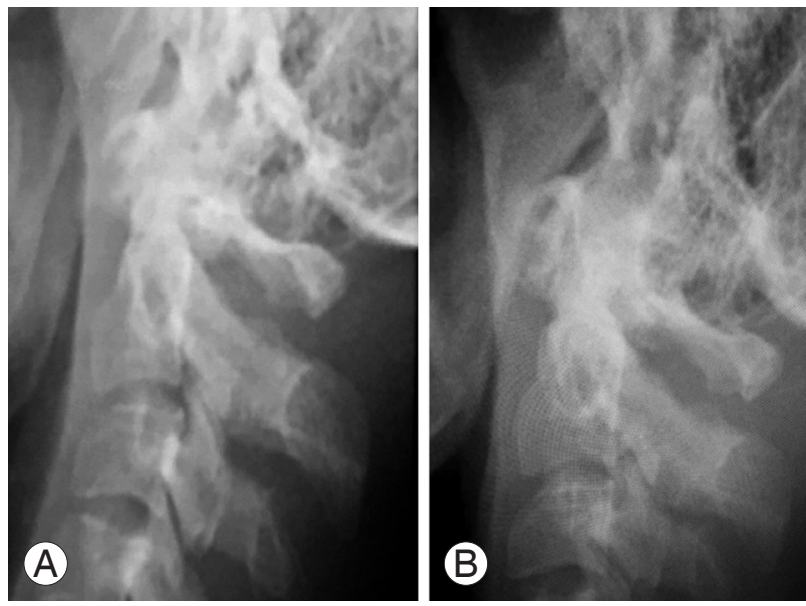

Fig. 5. Follow-up radiographs showing normal (A) flexion, and (B) extension in cervical spine.

C1-C2 segment was done with left C1 lateral mass screw and left $\mathrm{C} 2$ pedicle screw with technique as described in the literature (Fig. 3) [9]. He had an uneventful recovery and was discharged on postoperative day 3. Patient was immobilized in a rigid collar postoperatively for 2 weeks. Collar was discontinued in 2 weeks and patient was followed up in the office at 6 weeks, 3 months, and 6 months. CT scan was repeated at 6-month follow-up visit which showed healed odontoid fracture and excellent C1-C2 alignment (Fig. 4). At 9-month postoperative visit, patient was admitted for removal of internal fixation and discharged on postoperative day 1. Patient had normal flexion/extension and rotation of cervical spine at 1-year follow-up (Fig. 5).

\section{Discussion}

There is no universally accepted method of management of odontoid fractures $[11,12]$. The treatment of odontoid fractures could be considered conservative or surgical, depending on factors such as age, presence of associated 
injuries, and comorbidities [2,11]. Several factors like fracture healing, stability and residual stiffness need to be taken into consideration by the treating surgeon $[13,15]$. Fracture-related factors are related to the type of fracture, position of the fracture line, soft tissue injury, and presence of occipito-atlantal instability $[10,16]$. Hence, initial evaluation of a suspected cervical spine injury should include three plain radiographic views (i.e., AP, cross-table lateral, open-mouth odontoid). Sagittal, coronal, and axial CT reconstructions allow for characterization of fracture patterns, displacement, associated soft-tissue swelling and diagnose combined C1-C2 injuries. Measurement of fracture displacement and angulation on $\mathrm{CT}$ is critical in the treatment algorithm for odontoid fractures. MRI is indicated in the presence of a neurologic deficit and is considered the reference standard in the evaluation of the ligament injuries $[1,12]$. Evaluation of integrity of the transverse atlantal ligament (TAL) on the MRI directs the approach for internal fixation.

Currently, conservative management of type II fracture involves early immobilization using a halo vest or a rigid cervical collar. HTV is associated with complications including pin loosing, pin site infection and loss of fracture position, etc. HTV is not only more invasive but also more bulky and cumbersome in nature [8]. The nonunion rate of odontoid fracture with the use of HTV is about $11.4 \%$ and complication rate is about $43.3 \%$ as reported in the literature [13]. Rigid cervical collars not only have a higher nonunion rate compared to HTV, as they provide less support, but there is a risk of sudden death for patients $[12,13]$.

Surgical treatment has advantages over conservative therapy since the injury is immediately stabilized and rigid fixation obviates the need for halo fixation $[2,4,5]$. Surgical management is indicated in patients with fracture gap of $>2 \mathrm{~mm}$, posterior displacement of $>5 \mathrm{~mm}$, angulation of $>11^{\circ}$, rupture of transverse ligament and associated instability, comminuted fracture of dens as they are at risk of nonunion $[1,2,4]$. An anterior surgical approach for the management of odontoid fractures has the potential advantage to maintain rotational motion at the atlantoaxial joint. However, this approach has several complications, including neurovascular injury, esophageal and pharyngeal perforation, and airway compromise $[4,5,10,16]$. Contraindications of anterior odontoid screw fixation are TAL disruptions (as seen on MRI), irreducible odontoid fracture, and comminuted odontoid frac- tures $[2,12]$. Disruption of transverse ligament requires C1-C2 fixation because repair of odontoid fracture would not address $\mathrm{C} 1-\mathrm{C} 2$ instability. In this case, the TAL was intact as seen on MRI, however, considering the angulation and fracture gap, the surgeon felt there was a high possibility of getting inadequate reduction. C1-C2 fusion techniques have been successfully used in these patients with a fusion rate of $>95 \%$, but are associated with almost $50 \%$ reduction in cervical rotation, and a $10 \%$ reduction in flexion/extension, with consequent severe impairment of the activities of daily living. Occipitocervical fusion was not discussed with the patient in this case as it leads to disabling restriction of neck motion $[11,14]$. Significant loss of rotation was not acceptable to the patient nor his parents in this case report. Hence, the treating surgeon decided to use temporary internal fixation as an internal splint. Bilateral fixation would have been a more rigid fixation of $\mathrm{C} 1-\mathrm{C} 2$ segment, however, the patient had unstable lateral mass as result of Jefferson's fracture. In addition, the vertebral artery was abnormally closer to the pedicle on the right, hence, the surgeon decided to opt for unilateral fixation only on left side only. At 6-month follow-up CT scan, complete bony union was seen suggested by bony trabeculae crossing the fracture site and absence of sclerotic borders adjacent to the fracture site. The internal fixation was removed at 9 months. Further follow-up visit showed normal range of motion in the cervical spine and no instability on radiographs. The goal of bony fusion, relief of pain and normal motion was achieved.

In conclusion, there is no superior technique in treatment of type II odontoid fractures. The current treatment option of temporary internal fixation can be an important tool in the armamentarium of the surgeon in treating type II odontoid fractures in young adults and children. This strategy avoids the complication and hassles of halo fixation and immobilizes the unstable $\mathrm{C} 1-\mathrm{C} 2$ segment without fusion. Removal of the internal fixation after healing allows restoration of the rotational motion. A larger study with longer follow-up would validate the outcome of this technique.

\section{Conflict of Interest}

No potential conflict of interest relevant to this article was reported. 


\section{References}

1. Hsu WK, Anderson PA. Odontoid fractures: update on management. J Am Acad Orthop Surg 2010;18:383-94.

2. Maak TG, Grauer JN. The contemporary treatment of odontoid injuries. Spine (Phila Pa 1976) 2006;31:S5360.

3. Hadley MN, Browner CM, Liu SS, Sonntag VK. New subtype of acute odontoid fractures (type IIA). Neurosurgery 1988;22:67-71.

4. Rizvi SA, Fredo HL, Lied B, Nakstad PH, Ronning P, Helseth E. Surgical management of acute odontoid fractures: surgery-related complications and longterm outcomes in a consecutive series of 97 patients. J Trauma Acute Care Surg 2012;72:682-90.

5. Ben Aicha K, Laporte C, Akrout W, Atallah A, Kassab G, Jegou D. Surgical management of a combined fracture of the odontoid process with an atlas posterior arch disruption: a review of four cases. Orthop Traumatol Surg Res 2009;95:224-8.

6. Mazur JM, Loveless EA, Cummings RJ. Combined odontoid and jefferson fracture in a child: a case report. Spine (Phila Pa 1976) 2002;27:E197-9.

7. Anderson LD, D'Alonzo RT. Fractures of the odontoid process of the axis: 1974. J Bone Joint Surg Am 2004;86:2081.

8. Strohm PC, Muller Ch A, Kostler W, Reising K, Sudkamp NP. Halo-fixator vest--indications and complications. Zentralbl Chir 2007;132:54-9.
9. Harms J, Melcher RP. Posterior C1-C2 fusion with polyaxial screw and rod fixation. Spine (Phila $\mathrm{Pa}$ 1976) 2001;26:2467-71.

10. Hart D. The challenge of managing type II odontoid fractures. World Neurosurg 2013;79:664-5.

11. Kim SK, Shin JJ, Kim TH, Shin HS, Hwang YS, Park SK. Clinical outcomes of halo-vest immobilization and surgical fusion of odontoid fractures. J Korean Neurosurg Soc 2011;50:17-22.

12. Lewis E, Liew S, Dowrick A. Risk factors for nonunion in the non-operative management of type II dens fractures. ANZ J Surg 2011;81:604-7.

13. Fulkerson DH, Hwang SW, Patel AJ, Jea A. Open reduction and internal fixation for angulated, unstable odontoid synchondrosis fractures in children: a safe alternative to halo fixation? J Neurosurg Pediatr 2012;9:35-41.

14. Molinari RW, Dahl J, Gruhn WL, Molinari WJ. Functional outcomes, morbidity, mortality, and fracture healing in 26 consecutive geriatric odontoid fracture patients treated with posterior fusion. J Spinal Disord Tech 2013;26:119-26.

15. Harrop JS. Type II odontoid fractures: what to do? World Neurosurg 2013;80:313-4.

16. Patel AA, Lindsey R, Bessey JT, Chapman J, Rampersaud R; Spine Trauma Study Group. Surgical treatment of unstable type II odontoid fractures in skeletally mature individuals. Spine (Phila Pa 1976) 2010;35(21 Suppl):S209-18. 